\section{Canine osteoarthritis and treatments: a review}

\section{Stephanie D. Bland \\ Southern Illinois University, Carbondale, IL, USA}

\section{Abstract}

Arthritis is a commonly occurring chronic illness in human and animals alike. Among all domestic and pet animal species, dogs suffer from arthritis more often because of excessive running or exercise, injury, and/or genetic predisposition. Presently, one in four of 77.2 million pet dogs in the United States are diagnosed with some form of arthritis. In dogs, osteoarthritis is more common than rheumatoid arthritis and pain is the number one observation. Osteoarthritis, also known as degenerative joint disease, is a slowly progressive inflammatory disease, which is characterized by degeneration of the cartilage, hypertrophy of bone at the margins, and changes in the synovial membrane, and that eventually results in pain and stiffness of joints. Alterations in joint structures, decreased flexibility, and severe pain ensues, due to lack of hydration and inflammation. Cells within the damaged joints release pro-inflammatory cytokines, which further the inflammatory process. This causes more breakdown of the cartilage collagen type II and proteoglycans, which results in a perpetual destructive cycle. This perpetuating cycle ultimately results in cartilage destruction, subchondral bone thickening, and synovial membrane inflammation. This review focuses on osteoarthritis, the disease, causes, treatments, and presents a glimpse of some new therapies under study.

\section{Introduction}

Osteoarthritis, also known as degenerative joint disease (DJD), is a chronic inflammatory joint disease, which causes pain/soreness, stiffness, swelling, and lameness due to the diminished cushion and changes in the synovial fluid. ${ }^{1-3}$ Osteoarthritis affects the entire synovial joint including the cartilage, synovial fluid, and bone. This disease is characterized by degeneration of the cartilage and soft tissues, hypertrophy of bone at the margins, and changes in the synovial membrane..$^{1-3}$ Mechanical stress is thought to induce changes in biochemical factors within affected joints, leading to articular cartilage degradation. ${ }^{4}$ The disease process limits the amount of protein, released from the cartilage's cells, to repair cartilage in the joints; this is referred to as pitting and fraying of cartilage. 5 This pitting and fraying results in the cartilage losing its elasticity and protective surface due to enzymatic cleavage of proteoglycans. ${ }^{6}$ As the cartilage continues to break down and deteriorate completely, it causes friction between the bones, which leads to inflammation, thickening of soft tissues, and loss of mobility of the joint. ${ }^{7}$ Trying to maintain its normal balance of injury and repair, as the cartilage wears away the joints begin to lose its normal shape and the space between the joints narrow. Osteophytes (spurs) formation begins where the ligaments and joint capsule attach to the bone. In addition, fluid filled cysts form and fragments of bone and cartilage can be found floating in the joint space. ${ }^{5}$ All of the changes in the joints and bones can cause pain, swelling, and the joint may even appear enlarged.

\section{Disease overview}

Osteoarthritis is a disease that has been described for over a hundred years. ${ }^{8}$ Currently there are about 27 million Americans diagnosed, but is expected to reach 67 million by $2030 .{ }^{9}$ Osteoarthritis is the most common form of arthritis in humans and in dogs. In almost every form of arthritis there is a loss of bone or cartilage that results in changes in the shape of joints. 10 There are three different types of joints; fibrous, cartilaginous, and synovial. Fibrous and cartilaginous joints consist of fibrous tissues or hyaline cartilages, which allow little or no movement. Synovial joints are made up of synovial fluid and dense irregular connective tissue, which creates a synovial joint capsule allowing the joints to freely move. ${ }^{3}$ The main focus will be on the synovial joint, especially the ball and socket (hip and shoulder) and hinge joints (elbow), because these joints are most commonly affiliated with osteoarthritis, especially in canines. ${ }^{3}$ The synovial fluid in the synovial joint capsule provides nutrients, lubrication, and a cushion for articular cartilage. ${ }^{1-3}$ Articular cartilage, which is composed of hyaline cartilage, is avascular tissue consisting of chondrocytes embedded within an extracellular matrix of collagens, proteoglycans, and non-collagenous proteins. Articular cartilage reduces friction and makes movement of the synovial joints painless. ${ }^{11}$ The hyaline cartilage, which has a high content of collagen type II, serves as a shock absorber by distributing pressure from the load over the subchondral bone. In healthy joints, there is a fine balance between injury and repair amongst chondroblasts and chondroclasts. ${ }^{5}$ However, in osteoarthritis this balance is disrupted by an overproduction of osteoblasts
Correspondence: Stephanie D. Bland, Southern Illinois University, 1205 Lincoln Drive, Carbondale, IL, 62901, USA.

Tel.: +1.618.550.9490.

E-mail: sbland@siu.edu

Key words: Canine; osteoarthritis; NSAID; nutraceutical.

Acknowledgements: the author would like to acknowledge Ramesh Gupta, DVM, Ph.D. for serving as a mentor and providing general support and David Lightfoot, Ph.D. for critical revisions.

Conflict of interest: the author declares no potential conflict of interest.

Received for publication: 30 March 2015.

Revision received: 20 April 2015.

Accepted for publication: 21 April 2015.

This work is licensed under a Creative Commons Attribution NonCommercial 3.0 License (CC BYNC 3.0).

CC Copyright S.D. Bland, 2015

Licensee PAGEPress srl, Italy

Veterinary Science Development 2015; 5:5931

doi:10.4081/vsd.2015.5931

that can cause pain and swelling. Osteoarthritis has multiple causes and risk factors; however, once the cartilage is lost, the joint fails. ${ }^{12}$

Osteoarthritis is a progressive disease that consists of four stages. In stage one of osteoarthritis, minor bone spurs begin to develop. The cartilage matrix begins to break down due to chondrocyte's metabolism being affected and increasing the production of matrix destroying enzymes, metalloproteinases (MMPs). The severity of cartilage lesions can be correlated with the levels of collagenase present (MMP-1). ${ }^{13}$ Cartilage lesions disrupt the function of cartilage, increasing friction and inflammation in the joints, resulting in pain. Stage two of osteoarthritis is considered the mild stage. This stage involves erosion of the bone due to the cartilage lesions. This can cause new bone growth, osteophyte, also called bone spurs, which affect normal joint movement. In this stage, proteoglycan and collagen fragments are released into the synovial fluid. ${ }^{10}$ In the adult dog, protecoglycan turnover is quicker (300 days) than estimated collagen turnover (120 years). Marked protecoglycan loss of articular cartilage is irreversible and results in joint degeneration. ${ }^{4}$ Stage three is considered moderate osteoarthritis. The cartilage, in-between the bones, thins out and loses cushion. The space between the bones is also narrowing, causing grinding between the adjacent subchondral bones. ${ }^{10}$ During stage three, symptoms are more severe and inflammation begins to occur. Production of synovial 
macrophages occurs, including MMP, cytokines (interleukin 1), and tumor necrosis factor-alpha. ${ }^{4,6}$ Once the synovial macrophages are produced they can destroy tissues by diffusing back into the cartilage and can also stimulate chondrocytes. The fourth and final stage of osteoarthritis is considered severe osteoarthritis. In this stage the joint space is dramatically reduced, the cartilage is almost gone, and joint mobility is reduced greatly. 4,10 Early diagnosis of osteoarthritis is key to prevent further damage to the joint and alleviate symptoms.

\section{Diagnosis of canine osteoarthritis}

Osteoarthritis is the most common type of arthritis in dogs and is the most common source of chronic pain in older dogs. ${ }^{7}$ This is due to the constant wearing away of the cartilage from dogs running, jumping, and other strenuous exercise. Arthritis commonly affects large breed dogs, i.e. German Shepherds, Labradors Retrievers, Siberian Huskies, and Rottweilers, more than small breed dogs. Prevalence of osteoarthritis can be as high as $20 \%$ in dogs more than a year old, with middleaged and older dogs being at higher risk. Dogs that are diagnosed with arthritis tend to be lethargic, have difficulty moving from a sitting or lying position, cracking joints, stiffness, muscle wastage, and visible pain. ${ }^{7}$ Diagnosing osteoarthritis in dogs begin with owners observing the pain and stiffness while the animal is running, walking, jumping, or rising from a lying or sitting position. Radiographic evidence, patient symptoms, and osteoarthritis risk factors such as age, gender, and body mass index, can all aid in predicting the risk of rapid, highly predictable joint degradation. During physical exams, the patient may show signs of pain, including whining, biting, or trying to move away. Radiographic evidence can show the breaking down of cartilage between bones and inflammation in the joints. By properly diagnosing patients with osteoarthritis, this will help establish a future plan to help ease pain, prevent further damage, and overall increase the quality of life.

\section{Canine hip dysplasia}

Along with osteoarthritis, dogs may also suffer from hip dysplasia, a form of osteoarthritis present in the ball and socket joints. Hip dysplasia can be a genetically inherited condition from improperly formed hip joints typically seen in large breed dogs. ${ }^{14}$ Dogs that suffer from inherited hip dysplasia, show signs within the first year and should be spayed or neutered to avoid passing this genetic tenden- cy to malformation to offspring. Bulldogs, St. Bernard's, Blood Hounds, and Boykin Spaniels are a few examples of breeds that are at a higher risk factor for developing hip dysplasia. Dogs can also be at risk for hip dysplasia if there is excessive weight gain during the early stages of growth, typically 3-8 months of age, and from putting excessive pressure on the hip joint from strenuous exercise. Hip dysplasia is caused from an abnormal development of the hip joint, leading to excess laxity in the hip joint. Laxity in the hip joint can cause stretching of the supporting ligaments, joint capsules, and surrounding muscles, leading to permanent damage to the anatomy of the hip joint. ${ }^{15}$ The permanent damage to the anatomy causes the poorly developed head of the femur to loosely fit into a shallow acetabulum. 16 Orthopedic Foundation for Animals (OFA) radiographs can also be done to diagnose hip dysplasia. According to the Orthopedic Foundation for Animals, OFA radiographs must be performed with the animal in dorsal recumbancy with rear limbs extended parallel. The stifles are rotated inward and the pelvis is symmetric. This type of radiograph allows veterinarians to assess how the femoral head fits into the acetabulum, which aids in the diagnosis of hip dysplasia.16

\section{Measuring joint mobility}

Osteoarthritis patients struggle with limited range of motion (ROM), a reduction in the ability to move one's joints. Pain, stiffness, and swelling, all symptoms of osteoarthritis, can hinder mobility. Measuring the range of motion can help identify what condition the articular surface, joint capsule, ligaments, and muscles, are in. ${ }^{17}$ Assessing the range of motion is widely used in human medicine and is becoming more popular in canine veterinary medicine, as more patients are being diagnosed with arthritis (Table 1). Universal Goniometry is a commonly preferred way to measure range of motion in humans and other species. ${ }^{18}$ A goniometer is an affordable, reliable, commonly used, non-invasive tool used to measure flexion and extension degrees of joint mobility in the forelimbs and hind limbs in canine, as well as humans during physical therapy sessions. When using a goniometer, place the tool over the fulcrum of the joint, aligning the stationary arm with the stationary line of the body. Move the desired joint, either flexed or extend, and follow the moving line of the body with the moving arm of the goniometer; look at the readings on the goniometer for the degree of range of motion.

\section{Erythrocyte sedimentation rate}

In addition, a multitude of blood tests can be used to determine the degree of inflammation in the joints from arthritis, aiding in the diagnosis. One test used to assess inflammation is the erythrocyte sedimentation rate test along with complete blood counts and chemistry panels. The erythrocyte sedimentation rate (ESR) test, also known as the sed rate, sedimentation rate, and Westergren sedimentation rate, is a quick and simple test that has been used for many years to detect inflammation associated with infections, autoimmune diseases, and arthritis. A Polish pathologist, Edmund Biernacki, invented the ESR test in 1897. In 1918, two Swedish pathologists, Robert Sanno Fahraeus and Alf Vilhelm Albertsson Westergren used sodium citrate-anticoagulant specimens. This method of the test is widely used today and known at the Westergren method. 19

Due to the ESR test not being specific, it is used in addition to other blood tests including c-reactive protein, antinuclear antibody (ANA), and rheumatoid factor. Typically, ESR tests are ordered when a condition or disease is suspected to cause some form of inflammation in the body. For example, people who suffer from arthritis may have an ESR test run to detect the amount of inflammation in the joints. ESR is the rate at which red blood cells sediment in a period of one hour. The test is performed by anticoagulated blood, typically in an ethylenediaminetetraacetic acid (EDTA) tube that is placed in an upright $150 \mathrm{~mm}$ tube, also known as a Westergren tube. After an hour, the rate at which the red blood cells have fallen is reported in millimeters of plasma per hour $(\mathrm{mm} / \mathrm{hr}) .^{20}$ Normal ranges for canine and

Table 1. Maximum joint range of motion in canine.

\begin{tabular}{llc} 
Joint & \multicolumn{1}{c}{ Extension } & Flexion \\
Shoulder & 142 degrees-ground & 125 degrees-ground \\
Elbow & 124 degrees-ground & 98 degrees-ground \\
\hline Carpus & 124 degrees-ground & 97 degrees-ground \\
Hip & 141 degrees-ground & 115 degrees-ground \\
\hline Stifle & 141 degrees-ground & 109 degrees-ground \\
Hock & 135 degrees-ground & 115 degrees-ground \\
\hline
\end{tabular}


feline ESR are listed in Table 2. The ESR test works by a precise balance of pro-sedimentation factors, specifically fibrinogen, and resisting sedimentation factors, such as the negative charge of erythrocytes. During a state of inflammation, the fibrinogen increased causing the red blood cells to stick together in a stacked pattern known as rouleaux. The stacked erythrocytes are denser and cause the cells to settle faster than normal. ${ }^{21}$

\section{Drugs and disease management}

Animals with osteoarthritis are treated with various approaches, involving invasive and non-invasive measures. The objectives in managing osteoarthritis include minimizing joint pain by reducing the inflammation and slowing the progression of the cartilage damage, resulting in increased joint flexibility and ultimately improving quality of life. To achieve these goals, a variety of conventional pharmaceuticals, experimental treatments, nutracetuicals and supplements, and life change, such as stem cell therapy, physical therapy with acupuncture, and weight loss and exercise programs.

\section{Conventional treatments}

\section{Non-steroidal anti-inflammatory drugs}

Pharmacological management of osteoarthritis includes steroidal or nonsteroidal anti-inflammatory drugs (NSAID). These drugs do not address the underlying issue; they just control pain and inflammation. NSAIDs work against prostaglandins, which are a family of chemicals that are produced by cells and promote inflammation. Their inflammation properties also result in pain, fever, and increased platelet clumping. 1,7 The cells that produce prostaglandins are called cyclooxygenase (COX). There are two forms of COX enzymes, COX-I produces prostaglandins that support platelet clumping and protect the stomach, and COX-II enzymes produce prostaglandins that are responsible for pain and inflammation. Since NSAIDs inhibit both forms of COX enzymes, NSAIDs can result in gastrointestinal side effects, including ulceration, vomiting, anorexia, melena, and abdominal pain. 1,17

Acetylsalicylic acid was the first NSAID to be used in modern medicine and still is widely used. Acetylsalicylic acid, despite its side effects, is commonly recommended in veterinary medicine for dogs that suffer from osteoarthritis due to it being relatively inex-

Table 2. Erythrocyte sedimentation rate normal ranges.

\begin{tabular}{lc} 
Species & Normal range $(\mathrm{mm} / \mathrm{hr})$ \\
Feline & $0-12$ \\
Canine & $0-5$ \\
\hline
\end{tabular}

pensive. However, studies have shown that it can decrease chondrocyte production of collagen and proteoglycans and can enhance cartilage degradation over time. ${ }^{1}$ Acetylsalicylic acid is also a unique NSAID in the fact that it prolongs blood clotting for 4-7 days. This makes in an ideal drug for preventing blood clots that cause heart attacks and strokes, rather than an osteoarthritis event.1,7 Since there are many problems associated with acetylsalicylic acid for osteoarthritis treatment, other NSAIDs are becoming more popular. The six types of NSAIDs that are commonly prescribed by veterinarians, other than acetylsalicylic acid, for osteoarthritis patients include: carprofen, deracoxib, etodolac, meloxicam, tepoxalin, and firocoxib. ${ }^{7}$

\section{Corticosteroids}

Corticosteroids and gluccorticosteroids, often referred to as steroids, may be lifesaving and certainly increase the quality of life of dogs and humans. ${ }^{22}$ Cortisone is a hormone that naturally occurs in the cortex of the adrenal gland. This is where the cortico prefix comes from. Corticosteroids are produced from the same chemical base that produces the sex hormones. ${ }^{23,24}$ Cortisol is naturally produced when an animal gets stressed; however, manmade cortisol is 5-6 times stronger than naturally produced cortisol. Any production, natural or drug induced, of cortisol has a negative feedback and slows or stops natural production. Suppression of naturally produced cortisol typically occurs within 12-48 hours and takes a few days to start the process back up. ${ }^{25}$ Stopping the use of steroids quickly can result in a withdrawal syndrome, which includes fatigue, joint pain, stiffness, tenderness, and fever. 26

Corticosteroids are the most used, and misused, pharmaceuticals in veterinary medicine. ${ }^{22}$ Steroids, generally in an oral tablet, are used for stress response, immune system issues, inflammation, nutrient metabolism, and maintaining electrolyte levels in the blood. ${ }^{22}$ Corticosteroids are a popular treatment plan for patients suffering from arthritis because they are extremely effective in relieving pain and inflammation. ${ }^{23}$ Steroids inhibit the production of arachidonic acid, which can stop the inflammation and stop the production of prostaglandins, similar to NSAIDs. ${ }^{26}$ However, when using steroids the body cannot separate the anti-inflammatory properties from the immunosuppressant properties. ${ }^{26}$ Therefore, low doses of steroids are used to suppress inflammation and high doses of steroids are used as immunosuppressants. ${ }^{22}$ Since steroids affect nearly all cells of the body, their benefits are widespread, however, their side effects can be long lasting and devastating. ${ }^{26}$ The side effects, which vary depending on the dose and duration of steroid use, include sore mouth, weight gain, osteoporosis, high blood sugar levels (diabetes), cataracts, insomnia, gastrointestinal bleeding and ulcers, suppressed immune systems, fluid retention, atherosclerosis resulting in increased risk of heart disease, and aseptic necrosis. To reduce the probability of side effects from steroid use, one must avoid using steroids on a daily basis and no longer than 34 months without re-evaluating organ functions. Due to the devastating side effects of steroid use, alternative medicine such as acupuncture, nutraceuticals, and therapy can be used to treat osteoarthritis patients.

\section{Experimental treatments}

\section{Insulin growth factor-I}

Insulin growth factor-I (IGF-I) is peptide produced by the liver that promotes growth by reaching the articular cartilage through the synovial fluid. IGF-I can also be synthesized by chondrocytes. ${ }^{27}$ This peptide is vital for childhood growth, but continues to have anabolic affects in adults. IGF-I is stimulated by growth hormones and helps cartilage maintain structural and functional integrity by inhibiting interleukin-1's ability to stimulate proteoglycan degredation. ${ }^{27}$ However, under malnutrition conditions, hormone and receptor insensitivity, and failure to downstream signaling, can affect growth and stimulate damage to cartilage health. Past studies have shown that IGF-I can be important to the development of osteoarthritis and osteoporosis due to IGF-I being abundant in the cartilage and bone; therefore, it is suggested that it can prevent cartilage damage and the progression of osteoarthritis. ${ }^{27}$ This is because IGF-I plays a role in the regulation and homeostasis of normal cell growth and cartilage. In adults, if damage occurs, i.e. tumors, this can lead to overgrowth of bony tissue, which can cause osteoarthritis. ${ }^{28}$ However, due to many inconclusive studies, more work needs to be done to understand the mechanism and to what extent IGF-I has on osteoarthritis. 


\section{Oral doxycycline}

Doxycycline, broad spectrum, bacteriostatic antibiotic, is commonly used for bacterial infections and to treat malaria. However, preliminary studies on animals have been conducted to study the effects of doxycycline on osteoarthritis. It is speculated that low dosages of tetracycline analogue, specifically doxycycline, can inhibit the MMPs, which play an essential role in cartilage degradation. Due to doxycycline being more lipid soluble it is able to penetrate areas such as the synovial joint, which in the main site of damage in dogs with osteoarthritis. In one study conducted by the Johns Hopkins' Arthritis Center (1995), oral administration of doxycycline showed to prevent narrowing of the knee joint in subjects. Studies have also shown that oral administration of doxycycline can reduce the severity of articular cartilage, which plays a vital role in the process of osteoarthritis. ${ }^{29}$ In Nganvonpanit et al., the therapeutic effect of oral doxycycline on canine hip osteoarthritis, by reducing the rate of joint pathology in osteoarthritis, was studied. Overall, after a sixmonth period, dogs showed significant signs of improvement in joint mobility, pain upon limb manipulation, lameness, and were able to bear more weight on their hips. Signs of improvement were shown as early as two months. ${ }^{30}$ However, the results were not consistent and further studies should be conducted at the therapeutic effects of oral doxycycline and canine osteoarthritis and lameness. In addition, the overuse of doxycycline can result in microbial resistance; therefore, long-term side effects and dosage recommendations need to be further studied.

\section{Sodium pentosan polysulfate}

Originally made for humans who suffer from interstitial cystitis, sodium pentosan polysulfate is a semi-synthetic, poysulphated polysaccharide that has anti-inflammatory and antiarthritis properties and is classified as a disease modifying osteoarthritis drug (DMAOD). Sodium pentosan polysulfate is structurally similar to glycosaminoglycans. Sodium pentosan polysulfate is also similarly structured to the anti-coagulant, heparin; however, does not have the same strength. Sodium pentosan polysulfate has been studied in Europe for over 40 years, but only recently has been combined with calcium to increase its anti-arthritic properties. ${ }^{31}$ These properties are due to it being able to stimulate chondrocytes to synthesis cartilage, stimulate synoviocyte biosynthesis, and inhibit degradation of cartilage matrix and acrachidonic acid, which promotes an inflammatory cascade. 32 Sodium pentosan polysulfate is recommended to be injected into the joint in 5-7 day intervals with provided three months of relief. ${ }^{32}$ Currently, it is leading experimental treatment for osteoarthritis in canines and other animals by inhibiting MMPs and maintaining the cartilage structure and biochemistry. 32 Other than mild gastrointestinal upset, not other side effects have been noted. Therefore being relatively safe, even at three times the recommended dose and having minimal side effects, sodium pentosan polysulfate is becoming a more popular alternative treatment for canine osteoarthritis. ${ }^{31}$ However, more in vivo studies need to be performed to evaluate the bioavailability of oral route versus intramuscular.

\section{Glycoaminoglycans: glucosamine and chondroitin sulfate}

As the body ages the production of glucosamine slows down; therefore, it is important to supplement glucosamine to avoid joint issues. ${ }^{37}$ Glucosamine (2-amino-2-deoxy-D glucose), the most abundant monosaccharide, is a naturally occurring compound composed of sugar and amino acids. Glucosamine has been used for nearly 40 years in human medicine. 25 It is strictly used as a dietary supplement in the United States, but is a regulated pharmaceutical throughout Europe.16 There are three different types of glucosamine; glucosamine sulfate, glucosamine hydrochloride, and $\mathrm{N}$-acetylglucosamine. However, glucosamine sulfate may be more effective for arthritis treatment because sulfate is needed to produce cartilage and the other two forms of glucosamine do not contain sulfates. ${ }^{25}$ Glucosamine supplements are extracted from crustacean exoskeletons or from fermentation of grains such as corn or wheat. ${ }^{21}$ Glucosamine is one of the most commonly used nutraceuticals, especially for arthritic patients, due to it being involved in the body's production of joint lubrication and shock absorption and maintaining healthy cartilage and joint function. ${ }^{25}$ Glucosamine is the precursor in the biochemical synthesis of glycosylated proteins and lipids, glycosaminoglycans. Glycosaminoglycans are a major component of joint cartilage and the extracellular matrix of articular cartilage. ${ }^{25}$ Glucosamine also aids in the rebuilding of damaged cartilage and is a building block for articular cartilage. ${ }^{25}$ Glucosamine has anti-inflammatory properties by inhibiting synthesis of degradation enzymes, increasing synthesis of extracellular matrix, and reduces apoptosis of articular chondrocytes. ${ }^{25}$ Glucosamine is also good for nail growth, tendons, skin, eyes, synovial fluid, ligaments, heart valves, and mucous secretions of the digestive, respiratory, and urinary tract. ${ }^{21}$ Glucosamine supplements have little to no side effects when used at the recommended dose; however, if taken above the recommended dose, it can cause damage to pancreatic cells and increase the risk of diabetes. Shortterm side effects include stomach upset, constipation, diarrhea, headaches, and rashes.16 In recent years, in a series of preliminary experiments, researchers have evaluated several nutraceuticals individually and in combination with several other supplements, and found that they are significantly effective in ameliorating arthritic pain.

Glucosamine supplements are often combined with chondroitin sulfate. Chondroitin sulfate, a type of gylcoaminogycan, addresses the disease process of arthritis by aiding in the repair of damaged connective tissue. Chondroitin sulfate is one of the most abundant glycoaminoglycans in joint cartilage, bones, tendons, cornea, and heart valves..$^{21}$ It is also beneficial to stress injuries, by keeping joints hydrating and protecting existing cartilage breakdown. ${ }^{21}$ Studies has theorized that supplementation of chondroitin sulfate will maximize blood circulation to subchondral bone and synovial joints. Chondroitin sulfate is vital for articular cartilage and joint structure because it can bind collagen fibrils and is used as a chondroprotective agent by inhibiting the degradations of cartilage matrix and synovial fluid. ${ }^{21}$ Supplementation of chondroitin sulfate is important because as the body ages, less chondroitin sulfate is produced and other glycoaminoglycans, such as keratin sulfate, are produced which predisposes the joint to osteoarthritis. In addition to the joint benefits, chondroitin sulfate supplements are noted to have up to $70 \%$ bioavailability when taken orally, this is significantly more than the bioavailability of other supplements and nutraceuticals. Overall, glucosamine chondroitin sulfate and other joint related glycosaminoglycans, seem to be relatively safe and do not display any long term side effects. Therefore, making glycoaminoglycans a popular alternative treatment for osteoarthritis in canines. ${ }^{21,25}$

\section{Stem cell therapy}

Stem cell therapy, acupuncture, and massage therapy are all becoming popular aliments used to treat dogs that suffer from osteoarthritis symptoms. While most osteoarthritic treatments are the same for humans and dogs, stem cell therapy is only available for dogs. When anti-inflammatory agents are no longer improving the quality of life for arthritic dogs, stem cell therapy can be the next option. First discovered in 2005, by Dr. Brian Voynick of the American Animal Hospital, this therapy is an option for dogs with osteoarthritis or hip dysplasia. Stem cells are platelet rich plasma that can help inhibit the inflammatory process and repair damaged tissue. ${ }^{33}$ Currently, several therapeutic regenerative strategies have investigated whether autologous mesenchymal stem cells (MSCs) have significant effects on regeneration and maintenance of articular cartilage. ${ }^{33}$ Stem cell 
therapy is based on the isolation of these cells from fat or bone marrow tissues and then after culture expansion, they are injected back to the patient's damaged joints. ${ }^{33}$ Veterinarians claim that harvesting stem cells from fat is less invasive than a spay. A positive aspect to this therapy is that it is the dog's cells and therefore the risk of rejection is lower. Mesenchymal stem cells are responsible for releasing anti-inflammatory chemicals, which are speculated to repair damage in the joint. ${ }^{33}$ However, little is known about the mode of action when injected into the damaged joint. While stem cell therapy is still relatively new and seems to have promising effects for treating and preventing osteoarthritis in canines, it is a relatively expensive treatment, averaging around $\$ 3,000$, and results may vary from individual and severity of osteoarthritis.

\section{Nutraceuticals and natural products}

Pharmaceuticals have a high risk of toxicity and adverse side effects, because of this; there is push for alternative treatments in the form of food supplements. A nutraceutical is defined as a food, typically plant based, which provides medical or health benefits including the prevention and treatment of a disease. ${ }^{13}$ Stephen DeFelice, MD, the founder and chairman of the Foundation for Innovation in Medicine, coined the word nutraceutical in 1989 from the words nutrition and pharmaceutical. ${ }^{34}$ However, the use of food supplements to treat diseases dates back to Hippocrates, the father of medicine, (460-377 BC) when he predicted the health benefits of foods. ${ }^{35}$ Since certain foods play an important role in maintaining normal functions in the human body, nutraceuticals are gaining popularity with health professionals and the public. Currently, there are over 470 nutraceuticals with documented health benefits. ${ }^{13,15}$ Nutraceuticals are classified into two types; traditional foods and non-traditional foods. Traditional food is defined as natural, whole food with new information about potential health qualities. For example, omega-3 fatty acids in salmon and other seafood help reduce undesirable cholesterols. Non-traditional foods result from agriculture, crop and animal breeding or adding nutrients and ingredients to boost traditional food's nutritional value. Examples include orange juice that is fortified with calcium; milk fortified with vitamins; crops fortified with vitamins, minerals, and omega 3 s. However, to date few focus directly on osteoarthritis.

Unlike pharmaceuticals, there are no FDA regulations for the health claims of nutraceuticals or non-traditional foods. ${ }^{13}$ Even though there are few regulations on the health claims of nutraceuticals, safety must be assured in advance. Therefore, extensive, independent, testing must be reported on a nutraceutical before health professionals recommend it to their patients. During the research process, nutraceuticals can be classified as potential or established nutraceuticals. Nutraceuticals provide a promising approach towards a particular health or medical benefits, while established nutraceuticals have multiple, independent, peer-reviewed, research reports backing up their claimed benefits. ${ }^{31,36}$

Herbal medicine is increasing its popularity in veterinary medicine. ${ }^{7}$ Popularity may be due to low cost and a belief it has minimal to no side effects. Herbal medicine is becoming a common treatment for mastitis occurrences, foot-and-mouth disease outbreaks, skin allergies, food poisonings, tympany, and expulsion of placentae. In the past, nutraceuticals were a common therapy for livestock in treating a variety of diseases including hepatitis, chronic heart disease, skin disorders, wounds, and arthritis. ${ }^{24}$ Some nutraceuticals affect the progression of arthritis by preventing degradation and enhancing the repair of joint cartilage 37

\section{Weight control, exercise, and physical rehabilitation}

When treating osteoarthritis the main goals are to reduce pain and inflammation, improve joint function, eliminate or control the cause of arthritis, and even halt the process. Treatment can either occur through therapy or through medication. Osteoarthritis is more common in overweight dogs, so by putting the dogs on a strict diet to promote weight loss it can decrease mechanical stress that is placed on the joints. Obesity, and inactivity, which leads to obesity, can cause the joint to wear away faster due to extra pressure that is exerted on a joint. 138 According to the Arthritis Foundation, for every pound gained, three pounds of pressure are added to the knees and six pounds of pressure are added to the hips.1,38 By incorporating a weight loss program into the treatment plan this can lower the amount of medication the dog will need to take. Females, older dogs, and specific breeds, such as Beagles, Dachshunds, Collies, and Labrador Retrievers, are more prone to obesity. Therefore, a diet plan should be enforced to prevent and control body weight. Diets should be high protein and low fat, with a negative energy balance. Along with strict dieting, a modified exercise plan should also be established for the dog. An exercise program can help in reducing weight while maintaining range of motion and muscle mass. Modified exercises, low-impact like walking or swimming, can also strengthen joint supporting structures, muscles, ligaments, tendons, and joint capsules. ${ }^{1,7}$ Animal hospitals and rehabilitation facilities are starting to promote underwater treadmill therapy. This provides exercise with the lowest possibly impact due to the dog being in water. Overall, to create a successful treatment program the pet owner must be committed and willing to learn, the diet and exercise must be monitored, and post-diet weight monitoring by a veterinary should be done on a monthly basis.

\section{Future perspectives}

Currently, osteoarthritis is treated or managed by invasive as well as noninvasive means. ${ }^{24}$ In the recent past, the treatment options for arthritis were typically NSAIDs given alone or in combination with other disease-modifying agents. NSAIDs (COX enzymes inhibitors) eliminate pain, but do not eliminate the signs and symptoms of active disease nor do they repair cartilage. In recent years, chronic use of NSAIDs has been linked to numerous side effects, including gastrointestinal (GI) bleeding, and renal and hepatic dysfunction. Anti-inflammatory drugs such as aspirin and ibuprofen are non-specific inhibitors of COX enzymes (COX-I and COXII). ${ }^{37}$ They inhibit the production of inflammatory prostaglandins, resulting in their therapeutic effect, but also inhibit the production of constitutive prostaglandins, resulting in side effects, such as GI bleeding. ${ }^{37}$ Therefore, under these circumstances, a safe therapy is warranted for arthritic dogs. Nutraceuticals are also gaining popularity due to being readily available, inexpensive, and having minimal to no side effects. ${ }^{34,37-41}$ Nutraceuticals, such as type-II cartilage, shilajit, 5-loxin, avocado/soybean unsaponifiables, and curcumin have gained immense popularity for their antiarthritic and anti-inflammatory uses in humans and animals. However, with alternative medicine, there is no guarantee that the condition will improve; therefore, further safety and efficiency tests need to be performed to ensure the quality of these new treatments.

\section{References}

1. Vaughn-Scott T, Taylor JH. The pathophysiology and medical management of canine osteoarthritis. J S Africa Assoc 1997;68:215.

2. Lennon E, Marcellin-Little D. Canine osteoarthritis. 2005. Available from: http://www.arthritismd.com/canineosteoarthritis.html. Accessed January 2014. 
3. Pasquini C, Spurgeon T, Pasquini S. Anatomy of domestic animals stemic and regional approach. 11th ed. Sudz: Pilot Point; 2007.

4. Renberg WC. Pathophysiology and management of arthritis. Vet Clin Sm Anim 2005;35:559-64.

5. Scherer E. What happens in osteoarthritis? 2014. Available from: http://health. howstuffworks.com/diseases-conditions/ arthritis/osteoarthritis/what-happens-inosteoarthritis.html. Accessed January 2014.

6. Reid DM, Miller GC. Clinical trials in rheumatoid arthritis and osteoarthritis. Aberdeen, UK: Springer-Verlag London Limited; 2008.

7. WordPress. Osteoarthritis in dogs. Available from: http://www.osteoarthritisindogs.com/. Accessed January 2014.

8. Nelson AE, DeVellis RF, Renner JB. Quantification of the whole-body burden of radiographic osteoarthritis using factor analysis. Arthr Res Ther 2011;13:R176.

9. Iliades $\mathrm{C}$. The stages of osteoarthritis progression. 2014. Available form: http://www.everydayhealth.com/osteoarthr itis/stages-of-progression.aspx. Accessed January 2014.

10. Holland K. Stages of osteoarthritis of the knee. 2014. Available from http://www.healthline.com/health/osteoart hritis-stages-of-oa-of-the-knee?toptoctest=expand. Accessed January 2014.

11. Bos PK, Van Melle LM, Van Osch, GJVM. Articular cartilage repair and the evolving role of regenerative medicine. Open Access Surg 2010;3:109-22.

12. Eyre DR, Weis M, Wu J. Articular cartilage collagen: an irreplaceable framework? Eur Cells Mater 2006;12:57-63.

13. Rajat S, Manisha S, Robin S. Nutraceuticals: a review. Inter Res J Pharmacol 2012;3:95-9.

14. ASPCA. Hip dysplasia. 2014. Availabke from: http://www.aspca.org/pet-care/dogcare/hip-dysplasia. Accessed April 2014.

15. Tomiosso TC, Gomes L, De Campos Vidal B. Extracellular matrix of ostrich articular cartilage. Biocell 2005;29:47-54.

16. WebMD. Hip dysplasia in dogs: causes, symptoms, and tests. Availablr from: http://pets.webmd.com/dogs/hip-dysplasiadogs-causes-symptoms-tests. Accessed April 2014.

17. Lin $\mathrm{H}$, Shin F, Hou S. Digital imaging measuring of hip joint range of motion in dogs. Taiwan Vet J 2013;39:110-8.

18. Ates S, Hallaceli C, Hallaceli $H$. Goniometric measurements of the angular values of the joints in the fore- and hindlimbs of kangal dogs. Israel J Vet Med. 2011;66:166-70.

19. MedlinePlus. ESR. Available from: http://www.nlm.nih.gov/medlineplus/ency/ article/003638.htmMenon. Accessed August 2014.

20. AACC. ESR. American Association for Clinical Chemistry. Available from http://labtestsonline.org/understanding/an alytes/esr/tab/test/. Accessed August 2014.

21. Narcy SJ, Vangsness CT. Critical appraisal of the role of glucosamine and chondroitin in the management of osteoarthritis of the knee. Nutr Diet Suppl 2010;2:13-25.

22. Ward E. Steroid treatment. Long-term effects in dogs. VCA Animal Hospital. Available from: http://www.vcahospitals. com/main/pet-health-information/ article/animal-health/steroid-treatment-longterm-effects-in-dogs/951. Accessed February 2014.

23. Kidd R. Use corticosteroids on your canine with caution. Available from: h t t p : / / w w w. w h o l e - d o g journal.com/issues/7_7/features/Corticost eroids-For-Canine_5647-1.html. Accessed February 2014.

24. Mahima, Verma AK, Tiwari R. Nutraceuticals from fruits and vegetables at a glance: a review. J Bio Sci 2013;13:3847.

25. Simoens S, Laekeman G. Pharmacotherapeutic aspects of treating knee osteoarthritis with glucosamine sulfate. Health 2010;2:705-7.

26. Fields T. Steroid side effects: how to reduce corticosteroid side effects. Hospital for Special Surgery Journal. 2009. Available from: http://www.hss.edu/conditions_steroid-side-effects-how-to-reducecorticosteroid-side-effects.asp. Accessed February 2014.

27. Lloyd ME, Hart DJ, Nandra D, et al. Relation between insulin-like growth factor-I concentrations, osteoarthritis, bone density, and fractures in general population: The Chingford study. Amm Rheum Dis 1996;55:870-4.

28. Velloso CP. Regulation of muscle mass by growth hormone and IGF-I. Br J Pharm 2008;154:557-8.

29. Brandt KD. Modification by oral doxycycline administration of articular cartilage breakdown in osteoarthritis. J Rheum Suppl 1995;43:149-51.

30. Nganvongpanit K, Pothacharoen $P$, Suwankong N, et al. The effect of doxycycline on canine hip osteoarthritis: design of a 6-month clinical trial. J Vet Sci 2009;10:239-47.

31. Ghosh P. The pathobiology of osteoarthri- tis and the rationale for the use of petnosan polysulfate for its treatment. Semin Arthr Rheum. 1999;28:211-67.

32. Drugs.com. Cartophen vet (Canada) for animal use. Drugs.com. Available from: http://www.drugs.com/vet/cartrophen-vetcan.html. Accessed April 2015.

33. Franklin SP. Surgery STAT: stem cell therapy in canine medicine. DVM360. Available from: http://veterinarynews. dvm360.com/ surgery-stat-stem-cell-therapy-caninemedicine?pageID=3. Accessed April 2015.

34. D’Altilio MD, Peal A, Alvey M. Therapeutic efficacy and safety of undenatured type-II collagen singly or in combination with glucosamine and chondroitin in arthritic dogs. Tox Mech Meth 2001;17:189-96.

35. Singh P, Rani B, Chauhan AK. Healthy living with nurtaceuticals. Intern Res J Pharmcol 2012;2:12-4.

36. Sanghi D, Avasthi S, Srivastava RN. Nutritional factors and osteoarthritis: a review article. Inter J Med Up 2008;4:4253.

37. PetMD. Remedies for arthritis in dogs: glucosamine, chondroitin sulfate, steroids, and NSAIDs. Available from: http://www. petmd.com/dog/generalhealth/evr_dg_rem edies_for_arthritis_in_dogs?page $=2$. Accessed February 2014.

38. Suszynski M. Understanding primary and secondary osteoarthritis. 2014. Available from: http://www.everydayhealth. com/ osteoarthritis/primary-and-secondaryosteoarthritis.aspx. Accessed January 2014.

39. Deparle LA, Gupta RC, Canerdy TD. Efficacy and safety of glycosylated undenatured type-II collagen (UC-II) in therapy of arthritic dogs. J Vet Pharmacol Ther 2005;28:385-90.

40. Gupta RC, Canerdy TD, Lindley J. Comparitive therapeutic efficacy and safety of type-II collagen (uc-II), glucosamine and chondroitin in arthritic dogs: pain evaluation by ground force plate. J Anim Physio Anim Nutr 2011;96:770-7.

41. Gupta RC, Canerdy TD, Skaggs P. Therapeutic efficacy of undenatured typeII collagen (uc-II) in comparison to glucosamine and chondroitin in arthritic horses. J Vet Pharmacol Therap 2009;32: 577-84.

42. Millis DL. Canine rehabilitation and physical therapy. St. Louis: Saunders; 2004. p 536.

43. Provet. Hematology. 2014. Available from: http://www.provet.co.uk/geriatrics/5a65c32 .htm. Accessed August 2014. 